\title{
Consensus statement on the definition of neurogenic supine hypertension in cardiovascular autonomic failure by the American Autonomic Society (AAS) and the European Federation of Autonomic Societies (EFAS)
}

\author{
Endorsed by the European Academy of Neurology (EAN) and the European Society of \\ Hypertension (ESH)
}

\begin{abstract}
Alessandra Fanciulli ${ }^{1}$ - Jens Jordan ${ }^{2}$ - Italo Biaggioni ${ }^{3}$ - Giovanna Calandra-Buonaura ${ }^{4,5}$ - William P. Cheshire ${ }^{6}$. Pietro Cortelli ${ }^{4,5} \cdot$ Sabine Eschlboeck ${ }^{1} \cdot$ Guido Grassi $^{7,8} \cdot$ Max J. Hilz $^{9,10} \cdot$ Horacio Kaufmann $^{11} \cdot$ Heinz Lahrmann $^{12}$. Giuseppe Mancia ${ }^{13}$. Gert Mayer ${ }^{14}$. Lucy Norcliffe-Kaufmann ${ }^{11}$ • Anne Pavy-Le Traon ${ }^{15,16} \cdot$ Satish R. Raj $^{17}$. David Robertson ${ }^{3}$. Isabel Rocha ${ }^{18} \cdot$ Walter Struhal $^{19} \cdot$ Roland Thijs $^{20,21} \cdot$ Konstantinos P. Tsioufis $^{22} \cdot$ J. Gert van Dijk $^{21}$. Gregor K. Wenning ${ }^{1}$
\end{abstract}

Received: 20 February 2018 / Accepted: 24 April 2018 / Published online: 15 May 2018

(c) The Author(s) 2018

\begin{abstract}
Purpose Patients suffering from cardiovascular autonomic failure often develop neurogenic supine hypertension (nSH), i.e., high blood pressure (BP) in the supine position, which falls in the upright position owing to impaired autonomic regulation. A committee was formed to reach consensus among experts on the definition and diagnosis of $\mathrm{nSH}$ in the context of cardiovascular autonomic failure.

Methods As a first and preparatory step, a systematic search of PubMed-indexed literature on nSH up to January 2017 was performed. Available evidence derived from this search was discussed in a consensus expert round table meeting in Innsbruck on February 16, 2017. Statements originating from this meeting were further discussed by representatives of the American Autonomic Society and the European Federation of Autonomic Societies and are summarized in the document presented here. The final version received the endorsement of the European Academy of Neurology and the European Society of Hypertension.

Results In patients with neurogenic orthostatic hypotension, $\mathrm{nSH}$ is defined as systolic $\mathrm{BP} \geq 140 \mathrm{mmHg}$ and/or diastolic $\mathrm{BP} \geq 90 \mathrm{mmHg}$, measured after at least $5 \mathrm{~min}$ of rest in the supine position. Three severity degrees are recommended: mild, moderate and severe. nSH may also be present during nocturnal sleep, with reduced-dipping, non-dipping or rising nocturnal BP profiles with respect to mean daytime BP values. Home BP monitoring and 24-h-ambulatory BP monitoring provide relevant information for a customized clinical management.

Conclusions The establishment of expert-based criteria to define nSH should standardize diagnosis and allow a better understanding of its epidemiology, prognosis and, ultimately, treatment.
\end{abstract}

Keywords Neurogenic supine hypertension · Nocturnal hypertension · Neurogenic orthostatic hypotension $\cdot$ ABPM . Autonomic failure

Gregor K. Wenning

gregor.wenning@i-med.ac.at

Extended author information available on the last page of the article

\section{Introduction}

Primary cardiovascular autonomic failure develops in the context of inherited and sporadic neurodegenerative diseases affecting the autonomic nervous system. Secondary causes 
of cardiovascular autonomic failure include amyloidosis and metabolic or immune-mediated diseases inducing autonomic neuropathy [1].

The main feature of cardiovascular autonomic failure is neurogenic orthostatic hypotension $(\mathrm{nOH})$, defined in 2011 as a sustained reduction of systolic blood pressure (BP) of $\geq 20 \mathrm{mmHg}$ ( $\geq 30 \mathrm{mmHg}$ in patients with supine hypertension) or diastolic BP of $\geq 10 \mathrm{mmHg}$ within $3 \mathrm{~min}$ of standing or head-up tilt of at least $60^{\circ}$ [2]. About one-half of the patients with $\mathrm{nOH}$ develop neurogenic supine hypertension (nSH), which can be severe and last several hours during sleep [3]. $\mathrm{nSH}$ is distinct from essential hypertension, since most patients with $\mathrm{nSH}$ are normotensive while seated and may be severely hypotensive while standing [4-6].

As yet, no consensus criteria have been established for the diagnosis of $\mathrm{nSH}$, limiting current understanding of its epidemiology, prognostic significance and the development of appropriate management strategies.

To address this shortcoming, a round table discussion with participants from the European Federation of Autonomic Societies (EFAS) and from the American Autonomic Society (AAS) was convened in Innsbruck on February 16, 2017 to establish clinical criteria for the diagnosis of $\mathrm{nSH}$ in the context of cardiovascular autonomic failure.

Discussion points and statements originating from this meeting were subsequently examined and reviewed by representatives of both societies and are summarized in the document presented here. The final version was endorsed by the European Academy of Neurology (EAN) and by the European Society of Hypertension (ESH).

\section{Definitions}

\section{Supine hypertension}

In patients with proven $\mathrm{nOH}, \mathrm{nSH}$ is defined as systolic $\mathrm{BP}$ of $\geq 140 \mathrm{mmHg}$ and/or diastolic BP of $\geq 90 \mathrm{mmHg}$, measured after at least 5 min of rest in the supine position.

We propose the following ranges to define the severity of $\mathrm{nSH}$ in autonomic failure:

- Mild nSH: systolic BP values of $140-159 \mathrm{mmHg}$ or diastolic BP values of $90-99 \mathrm{mmHg}$.

- Moderate nSH: systolic BP values of 160-179 mmHg or diastolic BP values of 100-109 mmHg.

- Severe $\mathrm{nSH}$ : systolic BP values of $\geq 180 \mathrm{mmHg}$ or diastolic $\mathrm{BP}$ values of $\geq 110 \mathrm{mmHg}$.

\section{Nocturnal hypertension}

Patients with cardiovascular autonomic failure frequently show nSH also during sleep, i.e. nocturnal hypertension, with loss of the physiological nocturnal BP fall at night of $\geq$ $10 \%$ while supine and asleep (dipping). Two main pathological nocturnal BP profiles are distinguished:

- Reduced-dipping: characterized by a mean nocturnal BP reduction of $<10 \%$ with respect to mean daytime $\mathrm{BP}$ values.

- Non-dipping or rising: when the mean BP does not decrease or even increases during the night with respect to daytime $[7,8]$.

Additional aspects are important for an appropriate interpretation of $24 \mathrm{~h}$-ambulatory BP monitoring (24 h-ABPM) in patients with cardiovascular autonomic failure and are discussed in the section Diagnostic work-up.

\section{Clinical features}

Neurogenic supine hypertension is mostly asymptomatic or induces only non-specific complaints, such as headache. The main, most clinically relevant and immediate consequence of $\mathrm{nSH}$ is an exacerbation of pressure natriuresis during sleep, causing nocturia, sleep disturbances, volume depletion overnight and worsening of $\mathrm{nOH}$ in the morning $[9,10]$.

$\mathrm{nSH}$ could potentially result in hypertensive emergencies, with similar complications as in the general population, i.e. cerebral hemorrhage, ischemic stroke, acute pulmonary edema and myocardial infarction [11], although the overall occurrence of these clinical events has not been systematically investigated in cardiovascular autonomic failure. Acute cardiovascular complications associated with $\mathrm{nSH}$ have been reported, but mainly in patients also receiving anti-hypotensive drugs $[12,13]$, which are known to induce or exacerbate nSH (Fig. 1).

The long-term effects of cardiovascular autonomic failure have been studied in patients with Parkinson's disease (PD) and multiple system atrophy (MSA). Current evidence suggests that early-onset cardiovascular autonomic failure is associated with a poor prognosis [14-20], development of cardiovascular [21-23], kidney [24] and cerebrovascular [25-30] disease, as well as cognitive impairment [26, 27, 31-36]. Similarly, a higher prevalence of left ventricular hypertrophy, nephropathy and vascular and cerebrovascular disease has been reported in diabetic patients with secondary cardiovascular autonomic failure compared to those without [37].

At present, it is uncertain how much of the increase in risk is related to the underlying disease causing autonomic failure and how much is directly related to the abnormality in BP regulation. It is also unclear whether $\mathrm{nOH}$ or $\mathrm{nSH}$ represents the actual negative prognostic factor in patients with cardiovascular autonomic failure-or whether it is the BP volatility induced by the combination of both [38]. 
Fig. 1 Nocturnal blood pressure (BP) profiles at $24 \mathrm{~h}$-ambulatory BP monitoring a Physiological nocturnal dipping profile (mean nocturnal BP falls by $\geq 10 \%$ with respect to daytime BP), $\mathbf{b}$ reduced-dipping profile (mean nocturnal BP falls by $<10 \%$ with respect to daytime $\mathrm{BP}$ ), $\mathbf{c}$ rising profile (mean nocturnal $\mathrm{BP}$ increases with respect to daytime BP), in a patient with cardiovascular autonomic failure. Note severe hypotension occurring in the early morning. $H R$ Heart rate. The blue area indicates the pulse pressure, the green line indicates the mean BP. Adapted from Fanciulli et al. [44], with permission of Springer SBM. This image is excluded from the creative commons license
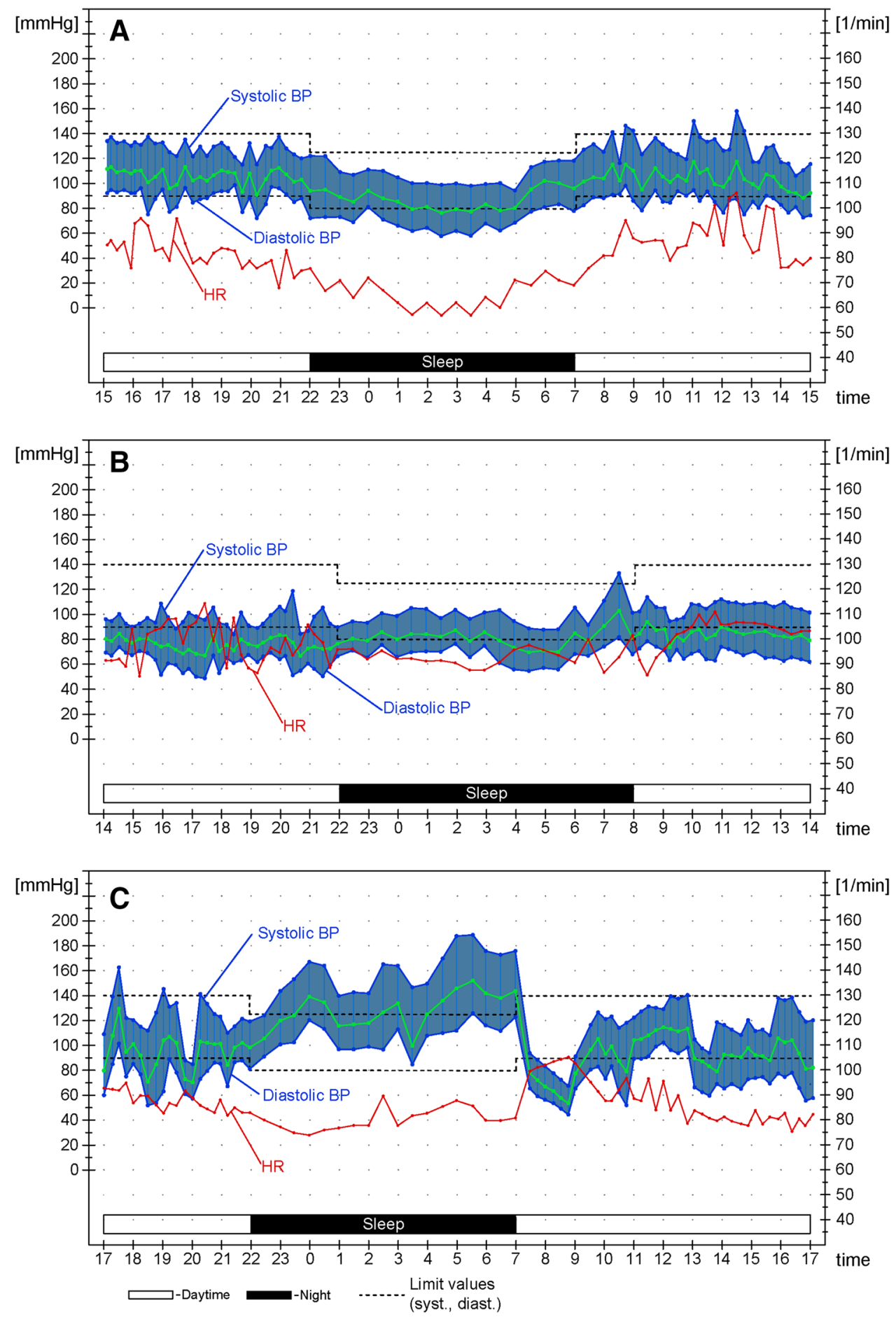

\section{Epidemiology}

Keeping in mind the limitations due to inconsistences in the diagnostic criteria, $\mathrm{nSH}$ has been reported in $34-46 \%$ of patients with PD and $37 \%$ of patients with MSA [3, 39]. The frequency rates of $\mathrm{nSH}$ increase to $50 \%$ in parkinsonian patients with $\mathrm{nOH}$, supporting the assumption of an etiological association between $\mathrm{nOH}$ and $\mathrm{nSH}[3,39-42]$. Loss of nocturnal BP dipping has been frequently reported in PD (48\%) and MSA patients (up to 75\%) [43-46].

The prevalence of nSH in pure autonomic failure (PAF) ranges from 48 to $70 \%$ [24, 47]. An abnormal, non-dipping, nocturnal BP profile has been described in up to $86 \%$ of patients with PAF [48].

To date, no studies have addressed the epidemiology of $\mathrm{nSH}$ in diabetes, but a significant association has been reported 
between the presence of $\mathrm{nOH}$ and rising nocturnal $\mathrm{BP}$ profiles both in patients with type 1 and type 2 diabetes mellitus [ 49 , 50].

The prevalence of nSH in genetic and acquired amyloidosis, as well as in other kinds of autonomic neuropathies is unknown. Clinically, patients with acquired amyloidosis (AL) and $\mathrm{nOH}$ often present with relatively low supine $\mathrm{BP}$ measurements which may be related to cardiovascular amyloid deposition.

\section{Pathophysiology}

Anti-hypotensive drugs used for the treatment of nOH may unmask or exacerbate $\mathrm{nSH}$, thereby posing a management challenge. However, $\mathrm{nSH}$ can also develop in the absence of anti-hypotensive treatment, which reflects the likelihood that multiple factors contribute to $\mathrm{nSH}$, including impairment of the afferent, central and efferent pathways of the arterial baroreflex arch, disruption of the renin-angiotensin-aldosterone axis and denervation supersensitivity at the level of the vascular adrenoceptors due to impaired sympathetic transmission [6, 41, 51-53].

Noradrenaline release from intact post-ganglionic sympathetic fibers in the setting of severe baroreflex impairment drives $\mathrm{nSH}$ in MSA, which is characterized in most cases by severe preganglionic sympathetic denervation, with sparing of the post-ganglionic sympathetic fibers to the heart and blood vessels [53].

In contrast, mechanisms independent of the sympathetic autonomic nervous system are considered to contribute to the development of $\mathrm{nSH}$ in autonomic disorders with post-ganglionic sympathetic denervation and very low circulating noradrenaline levels, such as in PAF [53]. Paradoxically increased angiotensin II and aldosterone receptor signaling in the setting of decreased activity of the systemic renin-angiotensin-aldosterone system has been implicated [51, 54]. In contrast, an impairment in nitric oxide-mediated vasodilation has not been observed in patients with $\mathrm{nSH}$ due to primary cardiovascular autonomic failure [55].

\section{Diagnostic work-up}

All patients newly diagnosed with $\mathrm{nOH}$ should be screened for $\mathrm{nSH}$ at the time of diagnosis and at regular intervals afterwards, especially if they begin treatment with antihypotensive agents, increase their dosage, report multiple episodes of nocturia per night or develop ankle edema.

Office screening should be performed by measuring supine BP as soon as the patient assumes the supine position and then again after the patient has been resting supine for at least $5 \mathrm{~min}$. This can be combined with a standing test, by having the patient stand immobile to avoid engaging the muscle pumps in the lower limbs, or with a tilt table examination. A tilt table examination may be preferred in patients unable to stand for several minutes.

It should be noted that the criteria outlined here to define $\mathrm{nSH}$ only apply for patients with proven $\mathrm{nOH}$ : if present, both conditions are usually verified during the same test. Exceptions to this rule may be due to the fact that $\mathrm{nOH}$ and $\mathrm{nSH}$ could manifest with different degrees of severity according to the time of the day, intravascular volume status and timing of the administration of anti-hypotensive drugs. Anti-hypotensive drugs may succeed in raising the standing $\mathrm{BP}$ and improving symptoms, but exacerbate $\mathrm{nSH}$.

A diagnosis of $\mathrm{nSH}$ should be also made independently from seated BP values, which may vary from hypertensive values to normo- or hypotensive values in patients with cardiovascular autonomic failure.

In addition to office BP screening, home BP recordings performed by patients themselves are recommended to gather further insights into circadian BP control. Although no protocol for home BP self-monitoring has been validated in patients with cardiovascular autonomic failure, we recommend that the BP be recorded three times per day (early morning, after lunch, at bedtime) in the supine, seated and standing position for 1 week at first diagnostic work-up. The same home BP protocol should be repeated after the initiation or adaptation of any treatment for $\mathrm{nOH}$ or $\mathrm{nSH}$ in order to monitor therapeutic effects and to detect potential side effects. Comparison of supine and seated BP values during the daytime is particularly valuable for choosing therapeutic strategies: patients with $\mathrm{nSH}$ may show normal BP values while seated, hence benefitting from a simple non-pharmacological approach such as avoiding the supine position during daytime.

If office BP screening or home BP recordings suggest $\mathrm{nSH}$, a $24 \mathrm{~h}-\mathrm{ABPM}$ is recommended to ascertain the presence of nocturnal hypertension and document absolute BP levels reached overnight. $24 \mathrm{~h}$-ABPM may also provide information on the severity of $\mathrm{nOH}$ during daily activity or after meals ("post-prandial hypotension" [56]), thus supporting customization of the therapeutic management [57].

Additional considerations are required when interpreting $24 \mathrm{~h}-\mathrm{ABPM}$ data of patients with cardiovascular autonomic failure:

- Several activities of daily life may cause severe drops in BP, which may be captured in the 24-h recordings. For an accurate interpretation of the 24 h-ABPM data, patients should be instructed to compile an activity diary on the examination day in which they report the times of medication intake (especially anti-hypotensive and anti-hypertensive agents), meals, physical activities and times of getting out of bed at night (for example, to use the bathroom). 
- Extreme drops in BP during daytime will reduce mean $\mathrm{BP}$ during the daytime, thereby resulting in an overestimation of the percentage of nocturnal BP rise. Conversely, nocturnal standing or bathroom visits may induce nocturnal BP falls, which may result in an underestimation of the BP rise overnight. For these reasons, both the percentage of nocturnal BP rise, as well as the absolute overnight BP values should be considered when diagnosing and tailoring treatment strategies for $\mathrm{nSH}$. Whereas non-dipping and rising nocturnal patterns have been associated with long-term end-organ damage, absolute BP values reached while supine may be more relevant for the development of hypertensive emergencies.

- Sleeping with the bed $12^{\circ}$ head-up tilted (or higher, if tolerated) creates an overnight gravitational stress and is an effective non-pharmacological strategy to manage nOH [58]. Sleeping with the whole bed tilted can mitigate the severity of nocturnal $\mathrm{nSH}$ by inducing venous pooling below the level of the heart throughout the night. Sleeping with only the head of the bed raised appears to be less effective in lowering night-time BP. Close attention should be therefore paid to the angle of the bed when interpreting the $24 \mathrm{~h}-\mathrm{ABPM}$ data.

- Patients with cardiovascular autonomic failure, especially in the setting of PD and MSA, may suffer from sleep fragmentation or sleep disordered breathing, both of which have been associated with development of hypertension [59-61]. If sleep disordered breathing is suspected at clinical history taking or based on $24 \mathrm{~h}$-ABPM data (for example, by documenting multiple nocturnal hypertensive peaks), a targeted diagnostic work-up is indicated.

- Nocturnal BP profiles may change over the short term with repeated $24 \mathrm{~h}-\mathrm{ABPM}$ in $10-35 \%$ patients with essential hypertension $[62,63]$. Reproducibility of the $24 \mathrm{~h}$-ABPM readings in patients with cardiovascular autonomic failure remains to be investigated. This may be important as BP can fluctuate on a day-to-day basis due to multiple factors.

Finally, if a nocturnal BP rise is documented in a patient not known to suffer from any autonomic disorder, screening for $\mathrm{nOH}$ may be indicated. Similarly, if a patient is found to have elevated BP while supine-for example, in a hospital or acute care setting - they should also have the BP measured seated and, if the systolic difference exceeds $10 \mathrm{mmHg}$, standing as well. Otherwise, $\mathrm{nSH}$ might be mistaken for essential hypertension, and if treatment decisions are based on supine BP values alone, medications could potentially worsen unrecognized $\mathrm{nOH}$.

\section{Perspectives}

Current epidemiological data are not sufficient to define cutoffs for $\mathrm{nSH}$ based on its impact on end-organ damage or mortality. For this reason, the criteria proposed here for the diagnosis of $\mathrm{nSH}$ in the context of cardiovascular autonomic failure are based on the criteria for the diagnosis of essential hypertension and interpretation of $24 \mathrm{~h}-\mathrm{ABPM}$ recordings in the general population $[7,8]$. There are several reasons supporting this strategy: first, to facilitate the use of these cut-off values in clinical practice; second, to apply severity degrees of $\mathrm{nSH}$ as a tool for customizing treatment and grade adverse events in future interventional clinical trials for $\mathrm{nOH}$; third, to generate data which can be compared across other epidemiological cohorts, such as essential hypertension. These definitions of $\mathrm{nSH}$ should ultimately lead to a better understanding of the prognostic outcome and stratification of the risk of adverse events.

Funding This was an academic project; no external financial support was allotted.

\section{Compliance with ethical standards}

Conflict of interest Alessandra Fanciulli: nothing to declare. Jens Jordan: served as advisor for Janssen-Cilag, Novartis, Novo-Nordisk and Theravance and received research support from Boston Scientific outside of the submitted work; he is also co-founder of Eternygen $\mathrm{GmbH}$. Italo Biaggioni: receives support from the NIH Rare Disease Clinical Research Network (U54-NS065736), reports consultancies for Lundbeck and Theravance for development of drugs to treat orthostatic hypotension and owns a patent application for an automated inflatable binder for the treatment of orthostatic hypotension. Giovanna Calandra-Buonaura: nothing to declare. William P. Cheshire: nothing to declare. Pietro Cortelli: nothing to declare. Sabine Eschlboeck: nothing to declare. Guido Grassi: nothing to declare. Max J. Hilz: nothing to declare. Horacio Kaufmann: receives support from the NIH Rare Disease Clinical Research Network (U54-NS065736). Heinz Lahrmann: nothing to declare. Giuseppe Mancia: nothing to declare. Gert Mayer: nothing to declare. Lucy Norcliffe-Kaufmann: receives support from the NIH Rare Disease Clinical Research Network (U54-NS065736). Anne Pavy-Le Traon: nothing to declare. Satish R. Raj: receives research support from the Canadian Institutes of Health Research (CIHR; Ottawa, ON, Canada) grant MOP142426 and the Cardiac Arrhythmia Network of Canada (CANet; London, ON, Canada) grants SRG-15-P01-001 and SRG-17-P27-001, and the Vanderbilt Institute for Clinical and Translational Research funded by a Clinical and Translational Science Award from the National Center for Advancing Translational Science from the National Institutes of Health (UL1 TR000445); he also reports consultancies for Lundbeck LLC and GE Healthcare. David Robertson: nothing to declare. Isabel Rocha: nothing to declare. Walter Struhal: nothing to declare. Roland Thijs: receives research support from the Dutch National Epilepsy Fund, ZonMW, NUTS Ohra Fund, Medtronic and AC Thomson Foundation, and has received fees for lectures from Medtronic, UCB and GSK. Konstantinos P. Tsioufis: nothing to declare. J. Gert Van Dijk: nothing to declare. Gregor K. Wenning: nothing to declare. 
Open Access This article is distributed under the terms of the Creative Commons Attribution 4.0 International License (http://creativeco mmons.org/licenses/by/4.0/), which permits unrestricted use, distribution, and reproduction in any medium, provided you give appropriate credit to the original author(s) and the source, provide a link to the Creative Commons license, and indicate if changes were made.

\section{References}

1. Benarroch EE (2014) The clinical approach to autonomic failure in neurological disorders. Nat Rev Neurol 10(7):396-407

2. Freeman R, Wieling W, Axelrod FB et al (2011) Consensus statement on the definition of orthostatic hypotension, neurally mediated syncope and the postural tachycardia syndrome. Clin Auton Res 21(2):69-72

3. Fanciulli A, Gobel G, Ndayisaba JP et al (2016) Supine hypertension in Parkinson's disease and multiple system atrophy. Clin Auton Res 26(2):97-105

4. Cicolini G, Pizzi C, Palma E et al (2011) Differences in blood pressure by body position (supine, Fowler's, and sitting) in hypertensive subjects. Am J Hypertens 24(10):1073-1079

5. Krzesinski P, Stanczyk A, Gielerak G, Piotrowicz K, Banak M, Wojcik A (2016) The diagnostic value of supine blood pressure in hypertension. Arch Med Sci 12(2):310-318

6. Goldstein DS, Pechnik S, Holmes C, Eldadah B, Sharabi Y (2003) Association between supine hypertension and orthostatic hypotension in autonomic failure. Hypertension 42(2):136-142

7. Parati G, Stergiou G, O'Brien E et al (2014) European Society of Hypertension practice guidelines for ambulatory blood pressure monitoring. J Hypertens 32(7):1359-1366

8. Mancia G, Fagard R, Narkiewicz K et al (2013) 2013 ESH/ESC guidelines for the management of arterial hypertension: the Task Force for the Management of Arterial Hypertension of the European Society of Hypertension (ESH) and of the European Society of Cardiology (ESC). Eur Heart J 34(28):2159-2219

9. Wilcox CS, Aminoff MJ, Penn W (1974) Basis of nocturnal polyuria in patients with autonomic failure. J Neurol Neurosurg Psychiatry 37(6):677-684

10. Jordan J, Shannon JR, Pohar B et al (1999) Contrasting effects of vasodilators on blood pressure and sodium balance in the hypertension of autonomic failure. J Am Soc Nephrol 10(1):35-42

11. Pinna G, Pascale C, Fornengo $P$ et al (2014) Hospital admissions for hypertensive crisis in the emergency departments: a large multicenter Italian study. PLoS One 9(4):e93542

12. Sandroni P, Benarroch EE, Wijdicks EF (2001) Caudate hemorrhage as a possible complication of midodrine-induced supine hypertension. Mayo Clin Proc 76(12):1275

13. Chaimberg KH, Travis KW (2002) Supine hypertension during general anesthesia in a patient taking midodrine. Anesth Analg 95(5):1196-1197 (table of contents)

14. Cilia R, Cereda E, Klersy C et al (2015) Parkinson's disease beyond 20 years. J Neurol Neurosurg Psychiatry 86(8):849-855

15. Stubendorff K, Aarsland D, Minthon L, Londos E (2012) The impact of autonomic dysfunction on survival in patients with dementia with lewy bodies and Parkinson's disease with dementia. PLoS One 7(10):e45451

16. CCalandra-Buonaura G, Guaraldi P, Sambati L, et al (2013) Multiple system atrophy with prolonged survival: is late onset of dysautonomia the clue? Neurol Sci 34(10):1875-1878

17. Petrovic IN, Ling H, Asi Y et al (2012) Multiple system atrophyparkinsonism with slow progression and prolonged survival: a diagnostic catch. Mov Disord 27(9):1186-1190
18. O'Sullivan SS, Massey LA, Williams DR et al (2008) Clinical outcomes of progressive supranuclear palsy and multiple system atrophy. Brain 131(Pt 5):1362-1372

19. Tada M, Onodera O, Ozawa T et al (2007) Early development of autonomic dysfunction may predict poor prognosis in patients with multiple system atrophy. Arch Neurol 64(2):256-260

20. Watanabe H, Saito Y, Terao S et al (2002) Progression and prognosis in multiple system atrophy: an analysis of 230 Japanese patients. Brain 125(Pt 5):1070-1083

21. Maule S, Milan A, Grosso T, Veglio F (2006) Left ventricular hypertrophy in patients with autonomic failure. Am J Hypertens 19(10):1049-1054

22. Maule S, Milazzo V, Maule MM, Di Stefano C, Milan A, Veglio F (2012) Mortality and prognosis in patients with neurogenic orthostatic hypotension. Funct Neurol 27(2):101-106

23. Vagaonescu TD, Saadia D, Tuhrim S, Phillips RA, Kaufmann H (2000) Hypertensive cardiovascular damage in patients with primary autonomic failure. Lancet 355(9205):725-726

24. Garland EM, Gamboa A, Okamoto L et al (2009) Renal impairment of pure autonomic failure. Hypertension 54(5):1057-1061

25. Oh YS, Kim JS, Lee KS (2013) Orthostatic and supine blood pressures are associated with white matter hyperintensities in Parkinson disease. J Mov Disord 6(2):23-27

26. Pilleri M, Facchini S, Gasparoli E et al (2013) Cognitive and MRI correlates of orthostatic hypotension in Parkinson's disease. J Neurol 260(1):253-259

27. Kim JS, Oh YS, Lee KS, Kim YI, Yang DW, Goldstein DS (2012) Association of cognitive dysfunction with neurocirculatory abnormalities in early Parkinson disease. Neurology 79(13):1323-1331

28. Gorell JM, Johnson CC, Rybicki BA (1994) Parkinson's disease and its comorbid disorders: an analysis of Michigan mortality data, 1970 to 1990 . Neurology 44(10):1865-1868

29. Tha KK, Terae S, Yabe I et al (2010) Microstructural white matter abnormalities of multiple system atrophy: in vivo topographic illustration by using diffusion-tensor MR imaging. Radiology 255(2):563-569

30. Lim TS, Lee PH, Kim HS, Yong SW (2009) White matter hyperintensities in patients with multiple system atrophy. J Neurol 256(10): 1663-1670

31. Jones JD, Jacobson C, Murphy M, Price C, Okun MS, Bowers D (2014) Influence of hypertension on neurocognitive domains in nondemented Parkinson's disease patients. Parkinsons Dis 2014:507529

32. Hohler AD, Zuzuarregui JR, Katz DI et al (2012) Differences in motor and cognitive function in patients with Parkinson's disease with and without orthostatic hypotension. Int J Neurosci 122(5):233-236

33. Allcock LM, Kenny RA, Mosimann UP et al (2006) Orthostatic hypotension in Parkinson's disease: association with cognitive decline? Int J Geriatr Psychiatry 21(8):778-783

34. Peralta C, Stampfer-Kountchev M, Karner E et al (2007) Orthostatic hypotension and attention in Parkinson's disease with and without dementia. J Neural Transm 114(5):585-588

35. Brown RG, Lacomblez L, Landwehrmeyer BG et al (2010) Cognitive impairment in patients with multiple system atrophy and progressive supranuclear palsy. Brain 133(Pt 8):2382-2393

36. Deguchi K, Takeuchi H, Sasaki I, Tsukaguchi M, Touge T, Nishioka M (2001) Impaired novelty P3 potentials in multiple system atrophy-correlation with orthostatic hypotension. J Neurol Sci 190(1-2):61-67

37. Milazzo V, Di Stefano C, Milan A et al (2015) Cardiovascular complications in patients with autonomic failure. Clin Auton Res 25(3):133-140

38. Rothwell PM (2010) Limitations of the usual blood-pressure hypothesis and importance of variability, instability, and episodic hypertension. Lancet 375(9718):938-948 
39. Umehara T, Matsuno H, Toyoda C, Oka H (2016) Clinical characteristics of supine hypertension in de novo Parkinson disease. Clin Auton Res 26(1):15-21

40. Shannon J, Jordan J, Costa F, Robertson RM, Biaggioni I (1997) The hypertension of autonomic failure and its treatment. Hypertension 30(5):1062-1067

41. Biaggioni I, Robertson RM (2002) Hypertension in orthostatic hypotension and autonomic dysfunction. Cardiol Clin 20(2):291301 (vii)

42. Pavy-Le Traon A, Piedvache A, Perez-Lloret S et al (2016) New insights into orthostatic hypotension in multiple system atrophy: a European multicentre cohort study. J Neurol Neurosurg Psychiatry 87(5):554-561

43. Berganzo K, Diez-Arrola B, Tijero B et al (2013) Nocturnal hypertension and dysautonomia in patients with Parkinson's disease: are they related? J Neurol 260(7):1752-1756

44. Fanciulli A, Strano S, Ndayisaba JP et al (2014) Detecting nocturnal hypertension in Parkinson's disease and multiple system atrophy: proposal of a decision-support algorithm. J Neurol 261(7):1291-1299

45. Plaschke M, Trenkwalder P, Dahlheim H, Lechner C, Trenkwalder C (1998) Twenty-four-hour blood pressure profile and blood pressure responses to head-up tilt tests in Parkinson's disease and multiple system atrophy. J Hypertens 16(10):1433-1441

46. Schmidt C, Berg D, Prieur S et al (2009) Loss of nocturnal blood pressure fall in various extrapyramidal syndromes. Mov Disord 24(14):2136-2142

47. Celedonio JE, Arnold AC, Dupont WD et al (2015) Residual sympathetic tone is associated with reduced insulin sensitivity in patients with autonomic failure. Clin Auton Res 25(5):309-315

48. Struhal W, Lahrmann H, Mathias CJ (2013) Incidence of cerebrovascular lesions in pure autonomic failure. Auton Neurosci 179(1-2):159-162

49. Madacsy L, Yasar A, Tulassay T et al (1995) Association of relative nocturnal hypertension and autonomic neuropathy in insulin-dependent diabetic children. Acta Biomed Ateneo Parmense 66(3-4):111-118

50. Chang J, Hou YP, Wu JL et al (2017) Blood pressure circadian rhythms and adverse outcomes in type 2 diabetics diagnosed with orthostatic hypotension. J Diabetes Investig 9(2):383-388

51. Arnold AC, Okamoto LE, Gamboa A et al (2016) Mineralocorticoid receptor activation contributes to the supine hypertension of autonomic failure. Hypertension 67(2):424-429
52. Arnold AC, Okamoto LE, Gamboa A et al (2013) Angiotensin II, independent of plasma renin activity, contributes to the hypertension of autonomic failure. Hypertension 61(3):701-706

53. Shannon JR, Jordan J, Diedrich A et al (2000) Sympathetically mediated hypertension in autonomic failure. Circulation 101(23):2710-2715

54. Arnold AC, Biaggioni I (2012) Management approaches to hypertension in autonomic failure. Curr Opin Nephrol Hypertens 21(5):481-485

55. Gamboa A, Shibao C, Diedrich A et al (2008) Excessive nitric oxide function and blood pressure regulation in patients with autonomic failure. Hypertension 51(6):1531-1536

56. Pavelic A, Krbot Skoric M, Crnosija L, Habek M (2017) Postprandial hypotension in neurological disorders: systematic review and meta-analysis. Clin Auton Res 27(4):263-271

57. Norcliffe-Kaufmann L, Kaufmann H (2014) Is ambulatory blood pressure monitoring useful in patients with chronic autonomic failure? Clin Auton Res 24(4):189-192

58. van Lieshout JJ, ten Harkel AD, Wieling W (2000) Fludrocortisone and sleeping in the head-up position limit the postural decrease in cardiac output in autonomic failure. Clin Auton Res 10(1):35-42

59. Gama RL, Tavora DG, Bomfim RC, Silva CE, de Bruin VM, de Bruin PF (2010) Sleep disturbances and brain MRI morphometry in Parkinson's disease, multiple system atrophy and progressive supranuclear palsy - a comparative study. Parkinsonism Relat Disord 16(4):275-279

60. Ghorayeb I, Yekhlef F, Chrysostome V, Balestre E, Bioulac B, Tison F (2002) Sleep disorders and their determinants in multiple system atrophy. J Neurol Neurosurg Psychiatry 72(6):798-800

61. Calandra-Buonaura G, Provini F, Guaraldi P, Plazzi G, Cortelli P (2016) Cardiovascular autonomic dysfunctions and sleep disorders. Sleep Med Rev 26:43-56

62. Cuspidi C, Meani S, Valerio C et al (2007) Reproducibility of dipping/nondipping pattern in untreated essential hypertensive patients: impact of sex and age. Blood Press Monit 12(2):101-106

63. Stenehjem AE, Os I (2004) Reproducibility of blood pressure variability, white-coat effect and dipping pattern in untreated, uncomplicated and newly diagnosed essential hypertension. Blood Press 13(4):214-224

\section{Affiliations}

\section{Alessandra Fanciulli ${ }^{1}$. Jens Jordan ${ }^{2} \cdot$ Italo Biaggioni $^{3} \cdot$ Giovanna Calandra-Buonaura $^{4,5} \cdot$ William P. Cheshire $^{6}$. Pietro Cortelli $^{4,5}$. Sabine Eschlboeck ${ }^{1}$. Guido Grassi ${ }^{7,8}$ - Max J. Hilz ${ }^{9,10}$. Horacio Kaufmann ${ }^{11}$ • Heinz Lahrmann ${ }^{12}$. Giuseppe Mancia ${ }^{13}$. Gert Mayer ${ }^{14}$. Lucy Norcliffe-Kaufmann ${ }^{11}$. Anne Pavy-Le Traon ${ }^{15,16}$. Satish R. Raj ${ }^{17}$. David Robertson ${ }^{3} \cdot$ Isabel Rocha ${ }^{18} \cdot$ Walter Struhal $^{19} \cdot$ Roland Thijs $^{20,21} \cdot$ Konstantinos P. Tsioufis $^{22}$ • J. Gert van Dijk ${ }^{21}$. Gregor K. Wenning ${ }^{1}$}

1 Department of Neurology, Innsbruck Medical University, Anichstraße 35, 6020 Innsbruck, Austria

2 German Aerospace Center and Chair of Aerospace Medicine, Institute of Aerospace Medicine, University of Cologne, Cologne, Germany
3 Division of Clinical Pharmacology, Vanderbilt University, Nashville, USA

4 Department of Biomedical and Neuromotor Sciences, University of Bologna, Bologna, Italy 
5 IRCCS, Institute of Neurological Sciences of Bologna, Bologna, Italy

6 Department of Neurology, Mayo Clinic, Jacksonville, USA

7 Clinica Medica, University of Milan-Bicocca, Milan, Italy

8 Istituto di Ricerca a Carattere Scientifico IRCCS Multimedica, Sesto San Giovanni, Milan, Italy

9 Department of Neurology, Universitätsklinikum Erlangen, Erlangen, Germany

10 Department of Neurology, Icahn School of Medicine at Mount Sinai, New York, USA

11 Department of Neurology, Dysautonomia Center, New York University School of Medicine, New York, USA

12 Private Practice, Vienna, Austria

13 Centro di Fisiologia Clinica ed Ipertensione, Milan, Italy

14 Department of Internal Medicine IV, Innsbruck Medical University, Innsbruck, Austria
15 Department of Neurology, French Reference Centre for Multiple System Atrophy, University Hospital of Toulouse, Toulouse, France

16 UMR INSERM 1048, Toulouse, France

17 Department of Cardiac Sciences, Libin Cardiovascular Institute of Alberta, University of Calgary, Calgary, Canada

18 Institute of Physiology, Faculty of Medicine, University of Lisbon, Lisbon, Portugal

19 Department of Neurology, Karl Landsteiner University of Health Sciences, Site Tulln, Tulln, Austria

20 Stichting Epilepsie Instellingen Nederland, Heemstede, The Netherlands

21 Department of Neurology, Leiden University Medical Centre, Leiden, The Netherlands

22 1st Department of Cardiology, Hippokration General Hospital, National and Kapodistrian University of Athens, Athens, Greece 